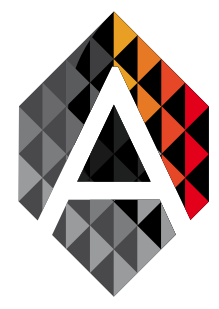

ADCAIJ: Advances in Distributed Computing and Artificial Intelligence Journal Regular Issue, Vol. 8 N. 4 (2019), 41-48 eISSN: $2255-2863$

DOI: http://dx.doi.org/10.14201/ADCAIJ2019844148

\title{
Detection of Hard Exudates in Retinopathy Images
}

\author{
Satya Bhushan Verma, Abhay Kumar Yadav \\ Department of Computer Science, BBA University (Central University), Lucknow, India \\ satyabverma1@gmail.com, abhaybbaucs01@gmail.com
}

\begin{tabular}{|c|c|}
\hline KEYWORD & ABSTRACT \\
\hline $\begin{array}{l}\text { Hard Exudates; } \\
\text { Retinopath; } \\
\text { Fundus Image; } \\
\text { Cotton Wool Spot; } \\
\text { Retina }\end{array}$ & $\begin{array}{l}\text { The tissue layer located at the back of the eye is known as retina which converts the } \\
\text { incoming light into nerve signals and those signals are sent to the brain for understand- } \\
\text { ing. The damage onto the retina is termed as retinopathy and that may lead to vision } \\
\text { weakening or vision loss. The hard exudates are small white or yellowish white deposits } \\
\text { with their edges being clear and sharp. In the proposed methods we take color image of } \\
\text { retina then extract the green channel of that image then apply top hat transformation and } \\
\text { bottom hat transformation on that image. The DIARETDB1 and High-Resolution Fun- } \\
\text { dus (HRF) databases are used for performance evaluation of the proposed method. The } \\
\text { proposed technique achieves accuracy } 97 \% \text {, sensitivity } 95 \% \text {, and specificity } 96 \% \text { and it } \\
\text { takes average } 5.6135 \text { second for detection of hard exudates in an image. }\end{array}$ \\
\hline
\end{tabular}

\section{Introduction}

Retina is the tissue layer located at the back of the eye that converts incoming light into nerve signals and then sent that signal to the brain for understanding. The damage onto the retina is called retinopathy which may lead to vision weakening or vision loss. Diabetes or hypertension is also detected by retinopathy. The retina's walls of blood vessel are thickened and narrow by constant high blood pressure. This places pressure on optic nerve and cause human vision difficulties. This type of condition is known as hypertensive retinopathy (Diana L. Shechtmanet al. 2007). If any signs of hypertensive retinopathy are present on the retina then it signifies the presence of soft exudates and hard exudates. Cotton Wool Spots (CWS) is also called soft exudates. Cotton wool spots are small, yellowish-white or grayish-white slightly elevated lessons which look like clouds on retina. CWS edges are blurry and not defined easily. The hard exudates are small white or yellowish white deposits with sharp margins.

Nowadays the role of image processing in automated identification of diseases in the retina is vital. It offers a non-invasive technique for the recognition of numerous retinal diseases like hypertensive retinopathy, diabetic retinopathy etc. Detected result will help us to take quick conclusion for the automatic appointments to the ophthalmologists. Detection of contributing signs of a diseased retina from the fundus image (Suma G. et al. 2018) will helps in early diagnosis of the disease and necessary treatment can be carried on further. Our proposed method tends to provide a quick and effective hard exudates detection technique. In United States the Diabetic retinopathy (DR) is one of the most common reasons of visual damage among working-age adults. The diabetic retinopathy happens when blood vessels are affected by diabetes in the retina (the light-sensitive 
tissue in the back of the eye), causing them to leak and distort vision. If this is not found and treated early, then diabetic retinopathy can causes permanent vision loss" (U. M. Akram et al. 2011).

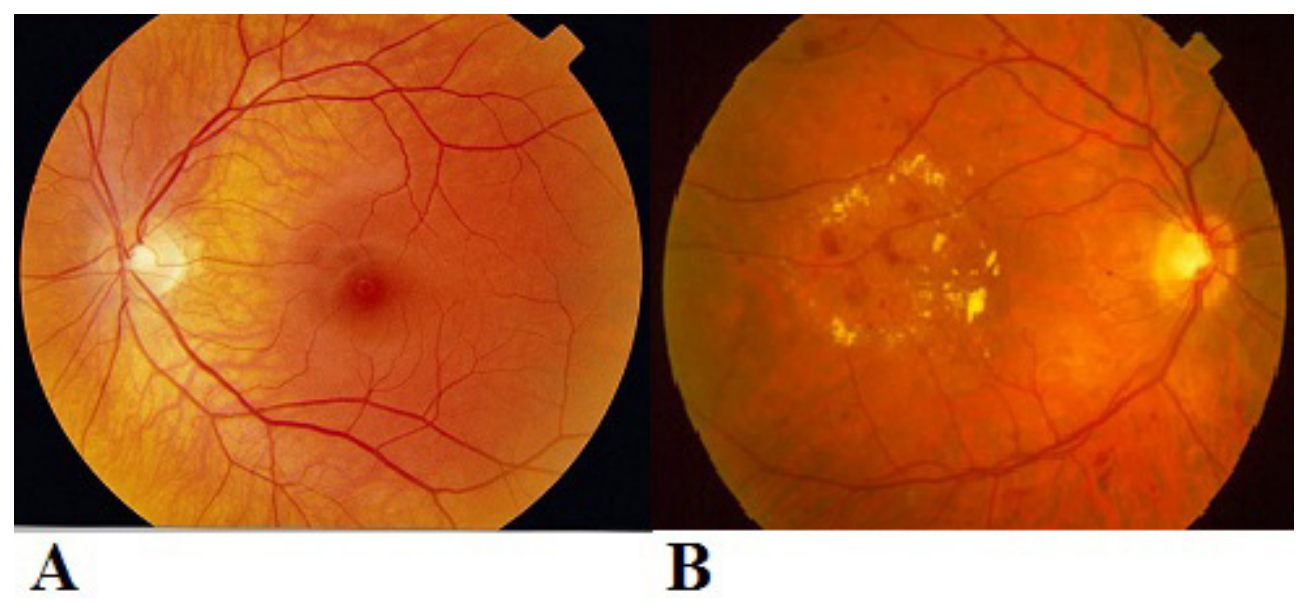

Figure 1: (A) Healthy retina (B) Retina with Hard Exudates.

The severity of Diabetic retinopathy is categorized based upon number of micro aneurysms, hemorrhages, exudates, and in neovascularization. The growth of Diabetic retinopathy is divided into normal retina, background DR, nonproliferative DR (NPDR), proliferative DR (PDR), and macular edema (ME). Diabetic retinopathy is a general microvascular problem. Diabetic macular edema is more common in type 2 diabetes, approximately $7.5 \%$ occurs in diabetic patients, and this is an important reason of blindness in working age person (Rajashekar D.et al. 2016).

\section{Literature Review}

Shengchun Long et al. (Shengchun L. et al. 2019) proposed an algorithm for Hard Exudates detection. Initially they make an automatic retinal image preprocessing method by using the active threshold technique and fuzzy C-means clustering (FCM) technique, then they used a classifier named support vector machine. Their proposed algorithm contains four stages first is preprocessing, second is localization of the optic disc, the third stage is the determination of candidate hard exudates by using dynamic threshold in the grouping with global threshold which is based on the FCM, and last stage is feature extraction. In the last stage, the candidate Hard Exudates region extracted the eight texture feature, which was then fed into an SVM classifier for the automatic Hard Exudates classification. They used DIARETDB1 (diaretdb1) and the e-ophtha EXretinal image database for the examination of their proposed algorithm. On the e-ophtha EX and DIARETDB1 retinal database they trained and cross-validated (10 fold) at the pixel-level. On e-ophtha EX database they achieved average sensitivity $76.5 \%$, PPV $82.7 \%$, and F-score $76.7 \%$, and on the DIARETDB1 retinal database. They achieved average sensitivity $97.5 \%$, specificity $97.8 \%$, and accuracy $97.7 \%$.

Iqbaldeep Kaur et al. (IqbaldeepK., et al. 2016.) managed to describe different works that were needed for the automatic identification of HEs and CWSs in retinal images for detection of diabetic retinopathy and images classification based on support vector machine (SVM). They evaluated their system on a large dataset having 129 retinal images. Their proposed method achieved sensitivity $96.9 \%$, specificity $96.1 \%$ and accuracy $97.38 \%$ for the detected exudates from a database.

Another interesting approach was proposed by Abhilash Goud Marupally et al (Abhilash G. M., et al. 2017) they put forward a Semi-automated quantification technique for detection of hard exudates in photographs of color fundus that were diagnosed with the diabetic retinopathy. Color fundus photographs of 30 eyes were taken from 30 subjects, 21 males and 9 females. They were able to develop a semi-automated algorithm to quantify 
the area covered by HEs. Two different methodologies were given (i) top-hat filtering, second order statistical filtering, (ii) color fundus images thresholding. They were able to detect $60-90 \%$ of the HEs area in 13 images and $90-100 \%$ in other 17 images.

Kavitha et al. (Kavitha, D. et al. 2005) uses the histogram based thresholding approach, wherein the local minima of the histogram were considered. They ignored the small fluctuations, and the last minimum was considered as the threshold value. This threshold value was applied to detect exudates with the optic disk of the eye. The blood vessels converge at the optic disk. Thus, the authors were able to find out the converging point of the blood vessels and the bright area that has kept this intersection point was considered as the optic disk, while the others were declared as exudates. An accuracy of $89 \%$, sensitivity of $92.87 \%$ and predictive value of 96.03\% was achieved.

Punnolil, A. et al (Punnolil, A. 2013.), proposed a simple thresholding method that can be used to extract the exudates by choosing an appropriate threshold level, their preprocessed image was complemented and had a threshold value of 0.97 , on which segmentation was performed. The binary image was then morphologically closed and opened for removal of blood vessels and optic disk, with a circular structuring element. Sensitivity of $96.89 \%$ and specificity $97.15 \%$ was achieved, but accuracy was not reported.

\section{Proposed Methodology}

The Proposed method involves following steps

\subsection{Image Acquisition}

The DIARETDB1 database [DIARETDB1] contains of 89 color fundus images, 84 images contain at least mild non-proliferative signs of the diabetic retinopathy, and the other 5 images are normal with no any signs of the diabetic retinopathy. The images were taken by the same 50-degree field-of-view using digital fundus camera with the varying imaging settings. High-Resolution Fundus (HRF) Image Database (High-Resolution Fundus (HRF)) contains total 45 images (15 images of healthy patients, 15 images of glaucomatous patients and 15 images of patients with diabetic retinopathy). Online databases with ground truth annotations are very important in helping researchers to compare the performance of different methods in medical imaging. Ground truth annotation are prepared first by computer vision researchers and then used by medical experts to select and annotate pathological signs (Kauppi T. et al. 2013.). This collaborative work creates ground truth images for computer-aided diagnosis systems.

\subsection{Image Preprocessing}

Image acquisition in retinal image mainly focuses on the OD area (nasal view) and the macular area In DIARETDB1, HRF, and local datasets, the captured images mainly focus on the macular area, which makes the illumination high at the macular area and makes the pixels at the outer ring of the image saturated (Rajashekar D.et al. 2016.). This might be the cause of image quality degradation and the presence of artifacts or noises during the process of image acquisition. Image preprocessing step is an essential step to improve the image quality (Verma, S. et al. 2019.). The green channel of RGB color space is selected because of its good contrast. Then the image enhancement and noise filtering are considered to enhance the contrast of the image and remove the noises respectively. 


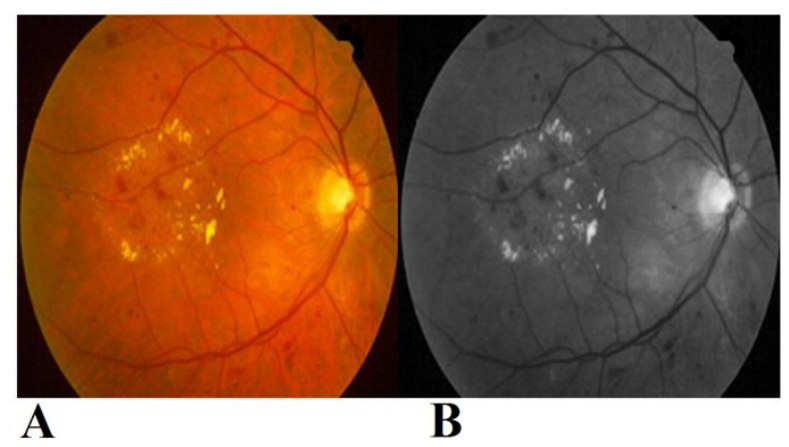

Figure 2: (A) Original Retinal Image (B) Retinal Image with green Channel Component.

\subsection{Image segmentation}

Image segmentation is one of the main steps in image processing techniques (Akram F, et al. 2017.). Several methods have been proposed to segment images based on attributes such as intensity, color, and texture. Thresholding is a useful image segmentation method and is frequently employed in medical imaging. It can be further classified into local thresholding and global thresholding.

\subsection{Morphological Top Hat Transformation}

The top-hat transformation is a process that removes small details from the images. There are two types of tophat transformation those are white top-hat transformation and black top-hat transformation (E. Aswini, et al. 2013.). The black top-hat transform is also known as bottom-hat transformation (A. Halder and P. Bhattacharya, 2015).

Let $f: E \rightarrow R$ be an image in grayscale,

Where, $\mathrm{E}$ is a mapping point from a discrete grid or Euclidean space. Let assume $b(x)$ be a grayscale structuring element.

Then, the white top-hat transform of $f$ is given by: $T_{t}(f)=f-f \circ b$, the black top-hat transform (bottom-hat transformation) of $f$ is given by: $T_{b}(f)=f \bullet b-f$.

Where

"o" denotes the opening operation.

"๑" denotes the closing operation.

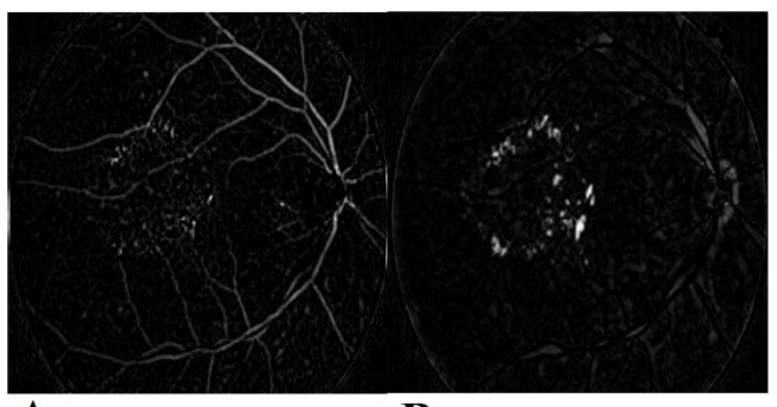

A

B

Figure 3: (A) fundus region after applying Morphological Bottom Hat (B) Fundus region after applying Morphological Top Hat. 


\subsection{Feature Extraction}

In the Proposed method top-hat transformation and bottom-hat transformation is used for the feature extraction.

$$
H e=T_{t}(f)-T_{b}(f)
$$

Where $T_{t}(f)$ is top-hat transformation and $T_{t}(f)$ is bottom-hat transformation.

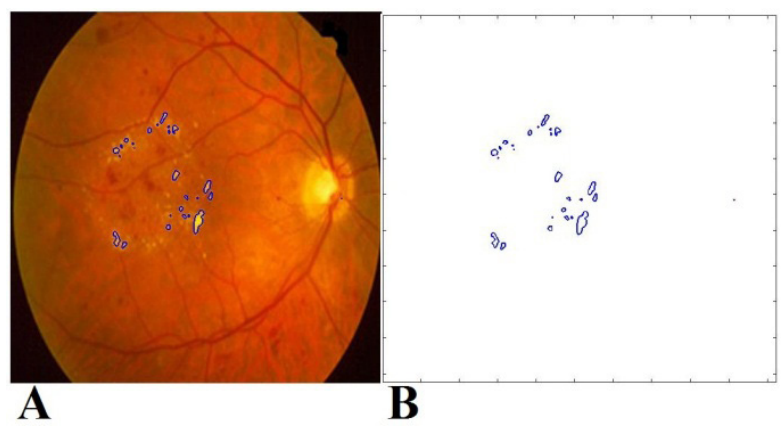

Figure 4: (A) Retina Image with Hard Exudates (B) Segmented Region of Hard Exudates.

\section{Experiment and Result}

High-Resolution Fundus (HRF) database and DIARETDB1 database are used for performance evaluation of the proposed method. Accuracy, sensitivity, and specificity are three performance metrics used for confirm the efficiency of the proposed method. Correctness of a diagnostic test is defined by accuracy (eq.2). The performance of positive disease detection is defined by sensitivity (eq.3) and specificity is known as chance of correct non-disease detection.

$$
\begin{aligned}
\text { Accuracy } & =\frac{T P+T N}{T P+T N+F N+F P} \\
\text { Sensitivity } & =\frac{T P}{T P+F P} \\
\text { Specificity } & =\frac{T N}{T N+F N}
\end{aligned}
$$

Where,

TP: Number of correctly classified Hard Exudates.

TN: Number of correctly classified Non-Hard Exudates.

FP: Number of wrongly classified Hard Exudates.

FN: Number of wrongly classified Non-Hard Exudates. 

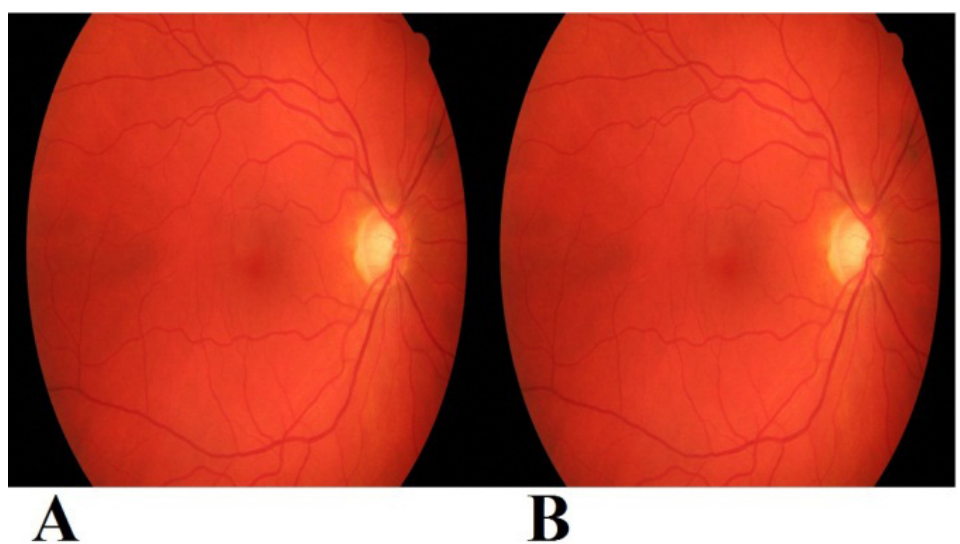

Figure 5: (A) Healthy retina (B) There is no any sign of Hard Exudates in retina.

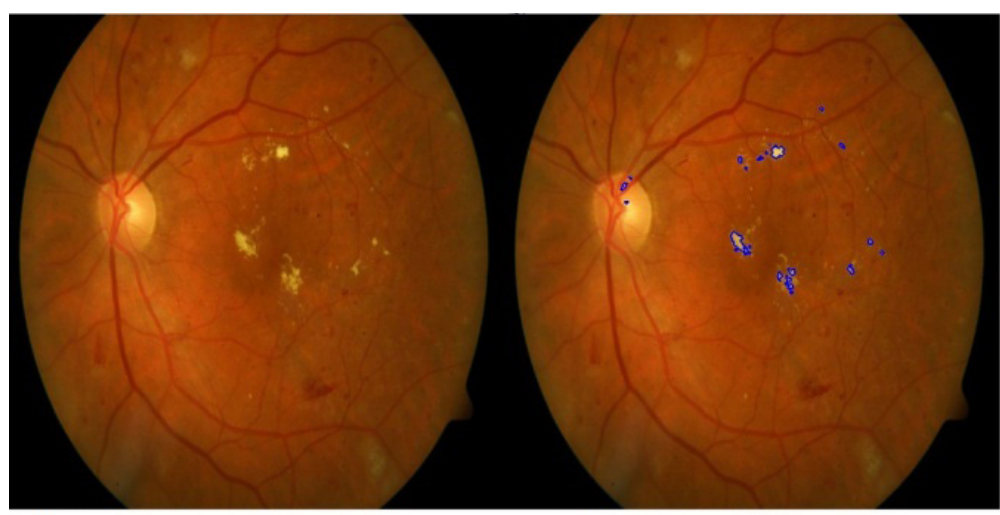

A

B

Figure 6: (A) Retina with Hard Exudates (B) Area of Hard Exudates in the retina.

The above figure 5(A) is an image of healthy retina and when this image passes with the proposed system resultant is figure 5(B) that clearly show there no any sign of hard exudates, but image in figure 6(A) passes through the proposed method then area of hard exudates is easily located. The following table1 shows the experimental results of the proposed method in the term of accuracy, sensitivity, and specificity those are $95 \%$, 94\%, 96\% in the DIARETDB1 database; and 97\%, 95\%, 95\% in the High-Resolution Fundus (HRF) database.

Table 1: Accuracy, Sensitivity, and Specificity in the DIARETDB1and High-Resolution Fundus (HRF) database

\begin{tabular}{|l|l|c|}
\hline \multicolumn{1}{|c|}{ Name of Database } & Performance Metrics & Results \\
\hline \multirow{3}{*}{ DIARETDB1 } & Accuracy & $95 \%$ \\
\cline { 2 - 3 } & Sensitivity & $94 \%$ \\
\cline { 2 - 3 } & Specificity & $96 \%$ \\
\hline \multirow{3}{*}{ High-Resolution Fundus (HRF) } & Accuracy & $97 \%$ \\
\cline { 2 - 3 } & Sensitivity & $95 \%$ \\
\cline { 2 - 3 } & Specificity & $95 \%$ \\
\hline
\end{tabular}


The experiment of the proposed model is carried out by using MATLAB R2015a on laptop with Intel core i3-23102.1 GHz processor and 4 GB RAM. The proposed method takes 5.6135 second for detection of hard exudates in retinopathy image. The proposed method is quite fast compared to other techniques.

\section{Conclusion}

The role of image processing is vital in the automated detection of diseases in retina. It provides a non-invasive methods for detection of many retinal diseases such as hypertensive retinopathy, diabetic retinopathy etc. In retina if any indication of hypertensive retinopathy then it contains the presence of soft exudates and hard exudates. Cotton wool spots (CWS) or soft exudates are small, light yellowish-white or grayish-white slightly higher lesions which appears as clouds on retina and their edges are blurry and not clearly defined. The hard exudates are small white or yellowish white deposits and their edges are clear and sharp. In the proposed methods we take color image of retina then extract the green channel of that image then apply top hat transformation and bottom hat transformation on that image. In the proposed technique we achieve accuracy $95 \%$, sensitivity $94 \%$, and specificity $96 \%$ in the DIARETDB1 database; and accuracy $97 \%$, sensitivity $95 \%$, specificity $95 \%$ in the High-Resolution Fundus (HRF) database. The proposed method takes average 5.6135 second for detection of hard exudates in an image.

\section{References}

A. Halder and P. Bhattacharya, 2015. An application of Bottom Hat transformation to extract blood vessel from retinal images in International Conference on Communications and Signal Processing (ICCSP), Melmaruvathur, pp. 1791-1795.

Abhilash G. M., et al. 2017. Semi-automated quantification of hard exudates in color fundus photographs diagnosed with diabetic retinopathy in BMC Ophthalmol 17, 172, doi:10.1186/s12886-017-0563-7

Akram F, et al. 2017. Active contours driven by local and global fitted image models for image segmentation robust to intensity inhomogeneity.PLoS One12(4):e0174813

Diana L. Shechtmanet al. 2007. Hypertension: More Than Meets the Eye in Review of Optometry, Vol.No: 144:09.

DIARETDB1 - Standard Diabetic Retinopathy Database,https://www.it.lut.fi/project/imageret/diaretdb1/index. html

E. Aswini, et al. 2013. Mathematical morphology and bottom-hat filtering approach for crack detection on relay surfaces in International Conference on Smart Structures and Systems - ICSSS'13, Chennai, pp. 108-113.

High-Resolution Fundus (HRF) Image Database, https://www5.cs.fau.de/research/data/fundus-images/

IqbaldeepK., et al. 2016. Automated Identification of Hard Exudates and Cotton Wool Spots using Biomedical Image Processing, International Journal of Computer Science And Technology, V. 7, I.4

Kauppi T. et al. 2013. Constructing benchmark databases and proto-cols for medical image analysis: Diabetic retinopathy.Computational and Mathematical Methods in Medicine, pp-1 - 15.

Kavitha, D. et al. 2005. Automatic detection of optic disc and exudates in retinal images, inInternational Conference on Intelligent Sensing and Information Processing, vol., no., pp.501-506.

Punnolil, A. 2013.A novel approach for diagnosis and severity grading of diabetic maculopathy in International Conference on Advances in Computing, Communications and Informatics (ICACCI), 2013, vol., no., pp.1230-1235, 22-25.

Rajashekar D.et al. 2016.Comprehensive retinal image analysis for aggressive posterior retinopathy of prematurity.PLoS One11(10)

Shengchun L. et al. 2019. Automatic Detection of Hard Exudates in Colour Retinal Images Using Dynamic Threshold and SVM Classification: Algorithm Development and Evaluation, in BioMed Research International, doi: 10.1155/2019/3926930 
Suma G. et al. 2018. Detection of Neovascularization in Proliferative, Diabetic Retinopathy Fundus Images, The International Arab Journal of Information Technology, 15, 1000-1009.

U. M. Akramet al. 2011. Automated Detection of Dark and Bright Lesions in Retinal Images for Early Detection of Diabetic Retinopathy, Springer Science Business Media, LLC. doi: 10.1007/s10916-011-9802-2

Verma, S. et al. 2019. Contactless palmprint verification system using 2-D Gabor filter and principal component analysis. The International Arab Journal of Information Technology.16(1), pp-23-29. 